\section{La universidad pública en la construcción de otra economía. Una perspectiva desde la Universidad Nacional del Centro de la Provincia de Buenos Aires}

\section{Marcos Pearson}

Coordinador del Programa de

Economía Social y Solidaria de la

Secretaría de Extensión, Universidad

Nacional del Centro de la Provincia

de Buenos Aires, Argentina.
Economía Social y Solidaria /

Desafíos de gestión

RECEPCIÓN: 26/06/15

ACEPTACIÓN FINAL: 29/07/15

\section{Resumen}

¿Cuál es el aporte fundamental que puede hacer la universidad pública para el desarrollo y fortalecimiento de una economía social y solidaria y cuáles son las principales limitaciones, desafíos y potencialidades que encontramos día a día en el proceso de construcción de otra economía?

El presente artículo tiene por horizonte compartir y exponer de forma ordenada algunas de las reflexiones y debates vinculados al desarrollo de la economía social y solidaria durante los últimos años en Argentina, poniendo especial atención a su presencia en el ámbito de las universidades públicas y de la articulación de éstas con las políticas públicas destinadas a su promoción.

Para exponer estas reflexiones y análisis se hará pie en la experiencia real y concreta que estamos desarrollando desde el año 2011 en el marco del Programa de Economía Social y Solidaria (Programa ESS) de la Secretaría de Extensión de la Universidad Nacional del Centro de la Provincia de Buenos Aires.

\section{Resumo}

Qual é a contribuição fundamental que pode fazer a Universidade pública para o desenvolvimento e fortalecimento de uma economia social e solidária e quais são as limitações, desafios e potencialidades mais importantes que nós achamos no dia a dia no processo de construção de uma "outra economia". Neste artigo procura-se compartilhar e expor de forma ordenada algumas reflexões e discussões relacionadas ao desenvolvimento da economia social e solidária nos últimos anos na Argentina, prestando especial atenção á sua presença no âmbito das universidades públicas e da articulação destas com as políticas públicas destinadas á sua promoção.

Para expor estas reflexões e análise se destacará a experiência real e concreta que nós estamos desenvolvendo desde o ano 2011 no contexto do Programa de Economia Social e Solidária (Programa ESS) da Secretaria de Extensão da Universidaed Nacional do Centro da Provincia de Buenos Aires.

Palavras-chave

- economia

- universidade

- autogestão

- trabalho

- equidade

Para citación de este artículo

Pearson, M. (2015). La universidad pública en la construcción de otra economía. Una perspectiva desde la Universidad Nacional del Centro de la Provincia de Buenos Aires. En Revista +E versión digital, (5), pp. 120-127. Santa $\mathrm{Fe}$, Argentina: Ediciones UNL. 


\section{Introducción}

Para poder realizar cualquier reflexión en torno a los alcances y desafíos que tiene por delante la universidad pública en su ineludible compromiso con el desarrollo de una economía social y solidaria (ESS), es necesario contextualizar y delimitar cómo es el escenario en el que se desarrolla actualmente, ya que este escenario es, en definitiva, el lugar desde donde partimos para todo lo que se pueda seguir avanzando en la dirección de una economía cada vez más justa, más humana y equitativa.

Por lo tanto, iniciaremos esta reflexión con un dato contundente: en Argentina, durante los últimos años, la ESS ha comenzado a ganar cada vez más lugar en la agenda pública.

Las prácticas y experiencias organizativas y laborales de diversos actores sociales - cooperativas y mutuales, movimientos campesinos y asociaciones de pequeños productores, emprendedores autogestionados, fábricas recuperadas, organizaciones de microcrédito, ferias y mercados populares, redes de comercio justo, radios y otros medios de comunicación comunitarios, etc.- que surgieron en gran medida como antídoto y respuesta frente a la crisis que explotó en el año 2001 encontraron un importante eco en el seno de nuestras comunidades. Es así que a partir de 2003 muchas de estas prácticas fueron reconocidas y promovidas por parte del Estado a través de diversas políticas públicas que están destinadas hacia su promoción, poniendo de manifiesto una forma distinta de abordar la temática del trabajo, la producción y las posibilidades de desarrollo de las personas. Este creciente reconocimiento que recae sobre los valores y las propuestas provenientes de la ESS indudablemente va de la mano de la capacidad de generar producción y trabajo digno que ha tenido y tiene en la actualidad este "movimiento" y del fuerte protagonismo que durante los últimos años cumplió el Estado, en sus diversas representaciones, en la puesta en valor de estas experiencias de organización, de trabajo y de consumo mediante una amplia batería de políticas públicas.

Con estos dos claros elementos que prefiguraron la reactualización y reconfiguración que la ESS vivenció durante la última década en Argentina - la organización social y popular y su articulación con el Estado y sus políticas públicas-arribamos a un estado de situación que se expresa cuantitativamente en los siguientes números: 27113 cooperativas, 4909 mutuales, 500000 monotributistas sociales, 311 empresas recuperadas, 250000 emprendimientos asociativos sin formalizar, 1600 organizaciones de microcrédito, 400000 productores de la agricultura familiar que producen el $70 \%$ de los alimentos que consumimos, 600 Ferias francas y de la ESS, 100 almacenes y mercados populares. ${ }^{1}$ Éste es, a grandes rasgos, el nivel de producción y de generación de trabajo que sostiene la ESS hoy en Argentina y que representa más de un millón de puestos de trabajo desarrollados entre 2003 y 2011 , lo que significa un aporte de aproximadamente el $10 \%$ del PBI del país.

\section{La economía social y solidaria en la Universidad}

En ese contexto, la presencia de la temática de la ESS en el ámbito universitario también es hoy una realidad con un reconocimiento innegable. Esto se manifiesta en distintas universidades en su creciente gravitación en los planes de estudios de las carreras de grado, en el desarrollo de maestrías y cursos de posgrado, en los programas y proyectos de extensión, en las líneas de investigación,
1) Estos datos fueron recopilados en base a información pública del Ministerio de Desarrollo Social de la Nación, el Instituto Nacional de Asociativismo y
Economía Social (INAES), el Instituto Nacional de Tecnología Agropecuaria (INTA), entre otros. 


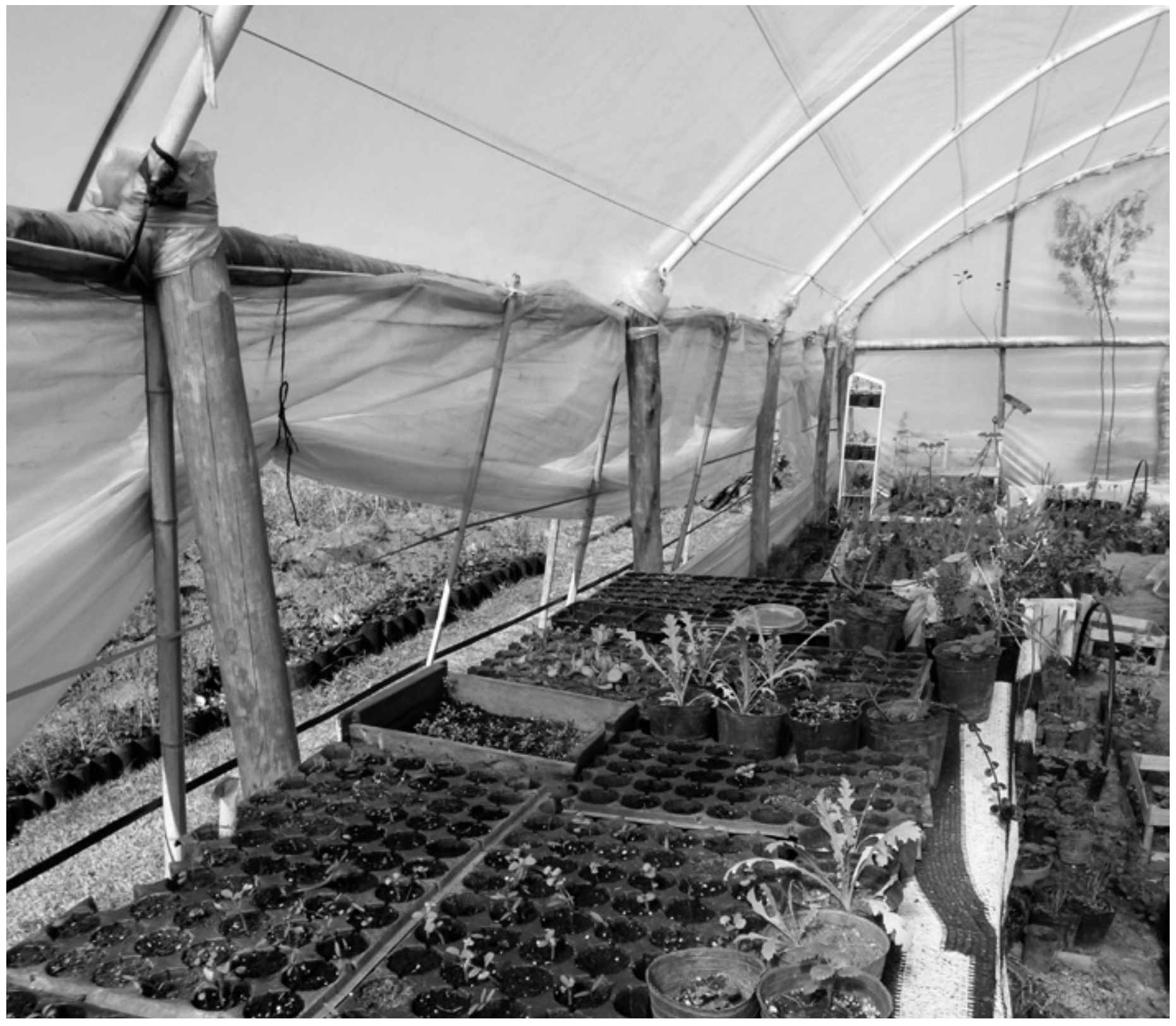


en la proliferación de seminarios, charlas y mesas debate en torno a la temática que se desarrollan desde hace más de diez años en las distintas universidades nacionales.

Un dato contundente y significativo que refuerza esta afirmación es la creación del Programa de Educación en Cooperativismo y Economía Social en la Universidad a través de la resolución 227 del año 2014 por parte de la Secretaría de Políticas Universitarias. A través del mencionado programa se están ejecutando actualmente más de 50 proyectos de investigación aplicada en distintos lugares del país que se llevan adelante de forma conjunta con organizaciones del sector y que tienen por horizonte la resolución de problemas reales y actuales para la ESS. ${ }^{2}$

Desde nuestra perspectiva, este alto grado de reconocimiento que las experiencias, prácticas y propuestas provenientes de la ESS han ido alcanzando dentro de la universidad pública representa una valiosa noticia de nuestro tiempo y, al mismo tiempo, un gran desafío. Es una valiosa noticia porque expresa un avance respecto de la institucionalización que va logrando el movimiento en ámbitos que durante largos años fueron colonizados y estuvieron reservados principalmente para el sentido hegemónico liberal y capitalista, como lo son las casas de altos estudios. Y es un gran desafío si pretendemos que esta presencia y reconocimiento se traduzca en un compromiso que se manifieste de forma integral por parte de la universidad en sus tres funciones esenciales -extensión, docencia e investigación- para el avance y la consolidación de un modelo económico que deje de ser pensado como un subsector de la economía y que la racionalidad que éste expresa - poner en el centro al ser humano antes que al afán de lucro como motor de la economía - sea la que ordene la sociedad de aquí en adelante. Hechos estos señalamientos y consideraciones introductorias compartimos ahora un breve acercamiento a la experiencia de trabajo que, en este campo, nos encontramos impulsando desde el Programa ESS de la Secretaría de Extensión de la Universidad Nacional del Centro de la Provincia de Buenos Aires (UNICEN) para, a partir de allí, poder compartir también algunas reflexiones en torno a las limitaciones encontradas en estos años y los principales desafíos que identificamos hacia adelante.

\section{El Programa de Economía Social y Solidaria}

El Programa de ESS de la Secretaría de Extensión de la UNICEN ${ }^{3}$ es una iniciativa que comenzó a trabajar en septiembre de 2011 con la intención de generar un espacio para la reflexión, la difusión y la acción con relación a la temática de la ESS en sus distintas dimensiones. Los principales objetivos del Programa de ESS son colaborar a la visibilización de la ESS en la región; impulsar acciones de capacitación, formación y fortalecimiento para emprendedores, productores y organizaciones de la ESS; contribuir a la formación de profesionales capaces de aportar sus saberes técnicos desde el compromiso con la construcción de la propuesta de la ESS; generar espacios de encuentro y trabajo colaborativo entre la Universidad, las organizaciones y movimientos sociales y el Estado como lugar de debate e intercambio de experiencias y como impulsor de la ESS como práctica ciudadana.

En este marco, el Programa desarrolla sus acciones dentro de los siguientes ejes de trabajo:

a) capacitación y formación;

b) comunicación y visibilidad de la ESS;

c) construcción de mercados en clave de ESS;

d) institucionalización de la ESS;

e) organización y fortalecimiento del sector;

f) proyectos específicos de Extensión Universitaria. 
De estos ejes se desprende una amplia batería de acciones. Dentro del eje de capacitación y formación, entre otras propuestas, se impulsa la cátedra de Cooperativismo y Economía Social y

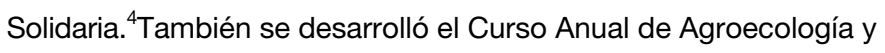
Economía Social y Solidaria, en un esfuerzo interinstitucional que involucró a distintas universidades nacionales, áreas y programas del Estado, así como a organizaciones sociales de la Agricultura Familiar y la Economía Social y Solidaria del país. De forma simultánea, y tanto dentro como fuera de la Universidad, se llevan adelante propuestas de formación a través de talleres y charlas con formatos más propios de la educación popular destinados a públicos diversos.

En el marco del eje comunicación y visibilidad de la ESS encontramos la participación en la elaboración del portal web Otra Economía Tandil, ${ }^{5}$ junto a otras acciones de visibilidad que se vinculan también con el eje de construcción de mercados, como lo es por ejemplo la experiencia del Circuito de Ferias de ESS en la ciudad de Tandil. ${ }^{6}$

El eje ligado a la institucionalización es el que encuadra la investigación y desarrollo de propuestas sobre marcos normativos adecuados a las prácticas de la ESS. En ese marco se impulsó un proyecto de ordenanza para la ESS en la ciudad de Tandil.

Finalmente, aunque no en orden de importancia, dentro del eje organización y fortalecimiento del sector, la Mesa de la Economía Social y Solidaria de Tandil, ${ }^{7}$ un espacio integrado por emprendedores, agricultores familiares, cooperativas, espacios de feria y comercialización e instituciones y organismos del Estado, constituye una de las principales apuestas de trabajo del Programa ESS.

\section{Limitaciones y desafíos en el desarrollo de la Economía Social y Solidaria en la Universidad}

Al igual que en la mayoría de las universidades públicas, la temática de la ESS ingresó institucionalmente en la UNICEN a través del campo de la extensión. Esto fue, como se ha señalado, a partir de 2011 con la creación formal de un Programa de Extensión en ESS alojado en la Secretaría de Extensión dependiente del Rectorado. En materia de extensión, podríamos decir que esto representa el grado máximo de institucionalización dentro de la Universidad. A eso se agrega que, dentro de la convocatoria interna a proyectos de extensión que la Universidad comenzó a impulsar desde el año 2012, se incorporó a la ESS como uno de los ejes prioritarios en virtud de lo cual se aprobaron y financiaron doce proyectos vinculados a la temática entre dicho año y 2015. Asimismo, cada uno de estos proyectos funciona vinculado al Programa, ámbito desde el cual se propicia el diálogo y el intercambio con los equipos integrantes de dichos proyectos, en la búsqueda de trazar, a mediano plazo y de forma participativa, las prioridades para la Universidad para el abordaje de esta temática en el territorio a nivel regional. Durante los primeros dos años de trabajo del Programa se priorizaron y enfocaron todos los esfuerzos en el involucramiento de la Universidad en el desarrollo de la ESS en la región. Esto se plasmó especialmente en la articulación con distintos organismos públicos, como por el ejemplo el Centro de Referencia del Ministerio de Desarrollo Social de la Nación, el Ministerio de Trabajo de la Nación, el Instituto Nacional de Tecnología Agropecuaria (INTA), las organizaciones de microcrédito, etc. En este contexto, se buscó promover la organización social del sector junto a emprendedores, productores y cooperativas con la convicción de que la construcción de herramientas colectivas y de participación real sería la única garantía para un trabajo de largo alcance y con profundidad social. Bajo esta lógica, y luego de un proceso de maduración, nació la Mesa de la Economía Social y Solidaria de Tandil —posteriormente se conformaron también en las ciudades de Azul y de Benito Juárez-, organización que continúa trabajando y en la cual el Programa de ESS participa activamente.

Sobre esta Mesa, como herramienta organizacional, el Programa de ESS volcó y continúa volcando muchos esfuerzos y expectativas y es a través de esta organización - que hoy involucra de forma activa a 40 compañeros y que se vincula con más de 400 emprendedores y productores familiares- que el Programa aborda y desarrolla algunos de sus ejes de trabajo. Por ejemplo, en el marco de la construcción de mercados está la propuesta del Circuito de Ferias de la ESS y, en breve, si todo avanza bien, un Almacén de la ESS dentro de la Universidad replicando la experiencia de la Universidad de Quilmes con su Almacén Autogestivo, Universidad con la cual tenemos un convenio de colaboración en estos temas. Por otro lado, respecto del eje de institucionalización de la ESS, el Programa impulsa activamente, junto a la Mesa ESS, un proyecto de ordenanza para el sector que actualmente se está discutiendo en el Concejo Deliberante de Tandil y que fue elaborado de forma participativa durante casi dos años a través de la realización de numerosos talleres, foros y mesas de debate. También se han impulsado y se continúan
4) Más información en: http://www. extension.unicen.edu.ar/economiasocial/?cat $=96$

5) Más información en: http://www. otraeconomiatandil.com.ar/ 6) Más información en: http://www. extension.unicen.edu.ar/economiaso$\mathrm{cial} /$ ?cat $=94$

7) Más información en: http://www. extension.unicen.edu.ar/economiaso$\mathrm{cial} /$ ?cat $=97$ 
impulsando propuestas de formación y capacitación en el marco de la Mesa y se desarrollan materiales de comunicación, difusión y sensibilización, entre otras tantas acciones.

Mientras se fue avanzando en este proceso e involucrados en estos proyectos, la primera limitación y preocupación que comenzamos a advertir tempranamente dentro del equipo del Programa ESS está vinculada a la articulación de estas prácticas extensionistas con las otras funciones de la Universidad.

Esto podría sintetizarse a través de las siguientes preguntas. Mientras la Universidad despliega en el territorio proyectos extensionistas en la dirección del fortalecimiento de la ESS, ¿qué tipo de profesionales se encuentra formando en cada una de sus unidades académicas? ¿Cuál es la presencia que tiene la temática en la currícula de las distintas carreras? ¿Cuáles son los temas y problemas que se priorizan desde el campo de la investigación? Las respuestas son múltiples y diversas conforme a cada campo y a cada unidad académica, pero lo que importa resaltar aquí es lo trascendental de abordar la temática de la ESS dentro de la Universidad de forma integral.

Tomassino y Rodríguez refieren a este desafío de la integralidad en el libro Tres tesis básicas sobre extensión y prácticas integrales en la Universidad de la República:

"La función de extensión no puede ser entendida como algo aislado, encapsulada en un gueto, sino que debe ponerse en interacción en el acto educativo y formar parte de manera naturalizada en el mismo. Este es un elemento central de apuesta por la integralidad pero que también presupone, que la investigación, también adquiera una relevancia mucho mayor en todos los espacios de la formación de los estudiantes y del trabajo docente; al igual que la extensión se debe naturalizar como una forma de aprendizaje" (2010:22-23).

Visualizando este desafío, identificamos que por más que realicemos muy buenos proyectos e iniciativas de cara a la comunidad acompañando a las cooperativas, o fortaleciendo la organización del sector, o promoviendo instancias de capacitación junto a emprendedores, productores y trabajadores autogestionados, el aporte y el compromiso de las universidades en el desarrollo de la ESS será siempre incompleto y de corto alcance si no problematizamos qué tipo de profesionales formamos en nuestras aulas y hacia dónde direccionamos nuestros esfuerzos en los equipos de investigación.
Esta tensión a resolver podemos advertirla con distintos ejemplos. En el campo de la producción de alimentos y el desarrollo rural, el sector de la agricultura familiar (AF) que produce y se organiza desde la perspectiva de la agroecología es una de las expresiones más claras de la economía social en el ámbito rural y periurbano. Los equipos extensionistas que trabajan en el fortalecimiento de la ESS desde hace mucho tiempo acompañan y trabajan codo a codo con pequeños productores y cooperativas de la $\mathrm{AF}$ intentando apuntalar a un sector que atraviesa grandes problemas para sostenerse y reproducirse en un mercado cada vez más concentrado y signado por el modelo del "agronegocio". En este contexto, ¿qué presencia tiene la perspectiva agroecológica en la formación de los ingenieros agrónomos en nuestras universidades? ¿Y en la investigación?

Los procesos de recuperación de fábricas por parte de sus trabajadores conformando cooperativas para defender sus fuentes laborales y poner nuevamente en marcha muchas empresas que quebraron o "fueron vaciadas" por sus propios dueños y que hoy en Argentina alcanzan a más de 300 cooperativas que involucran a 13462 trabajadores, ${ }^{8}$ representan, también, uno de los fenómenos más potentes de la ESS en Argentina de la última década. Expresan en su praxis misma, y en un mismo movimiento, las limitaciones propias de una racionalidad que pone en el centro al afán de lucro a cualquier costo y el potencial del trabajo autogestionado, asociativo y cooperativo. Dicho de otro modo, son experiencias que sintetizan de forma concreta la máxima que reza: "otra economía es posible". En muchos casos las universidades acompañamos estos procesos, pero cabe la pregunta: ¿cuántos contadores, administradores o abogados formados en nuestras casas de estudio están en condiciones de "asesorar" o participar con sus saberes profesionales en estas empresas? Más aun, ¿cuántos de nuestros profesionales conocen sobre la figura jurídica de las cooperativas y mutuales tanto como conocen de las otras figuras propias de las entidades con fines de lucro?

Ahora bien, este planteo crítico respecto de las limitaciones que encontramos en la universidad en el abordaje de la ESS debe ser leído siempre en clave de desafío y de proceso. Nos movemos en un cuadro institucional que tiene por herencia 30 años de profundización de una mirada neoliberal de la economía en el marco de una estrategia de colonización por parte del capital que fue de alcance regional y a la cual la universidad pública no escapó. Durante 30 años, entonces, nuestras sociedades sufrieron un 
aplastante avance del capital y la especulación financiera por sobre el trabajo, y de una cultura individualista y de consumo por sobre la solidaridad y el bien común. Esto obviamente también caló muy hondo en el campo educativo y ese es el proceso que, desde las universidades, se está intentando deconstruir al menos desde el año 2003 hacia acá.

Dentro de nuestra universidad, la reciente cátedra de Cooperativismo y Economía Social y Solidaria, que hoy está presente en el curriculum de cuatro de nuestras unidades académicas y que impulsamos por segundo año consecutivo con una importante participación del estudiantado; la creación de nuevos programas de extensión en ESS hacia el interior de distintas facultades; y los proyectos de investigación que actualmente estamos llevando adelante dan cuenta de que, luego de haber identificado el problema, vamos caminando en la dirección de esa integralidad en las funciones de la Universidad.

\section{Valorar los valores}

Lo último que quisiéramos señalar en cuanto a la ESS en su vinculación con la universidad está referido a uno de los mayores problemas que identificamos recurrentemente en el trabajo en la comunidad y a una posible estrategia para su resolución en donde las universidades pueden jugar un rol gravitante.

Identificamos, por un lado, en los números que se expusieron anteriormente, un enorme potencial de generación de trabajo digno y de capacidad producción de bienes y servicios dentro del sector de la ESS. Advertimos, por otro lado, un problema que se repite como un mantra para la mayoría de las experiencias de la ESS: la comercialización. Es decir, hay una gran capacidad de producción que tiene como correlato una gran dificultad para insertar esos productos y servicios en el mercado tradicional.
Esto representa un problema de fondo cuya estrategia abordaje de mediano y largo plazo podría estar ligada a dos ejes centrales: lo comunicacional y lo institucional.

En nuestro país, aunque desde el gobierno nacional se realizan desde hace largos años esfuerzos por revertir esta situación, contamos en los diversos rubros de la economía con mercados muy concentrados, atravesados por una altísima dinámica de intermediación y marcados por una lógica sumamente especulativa. En este contexto, es natural que los productos y servicios que se generan en la clave de ESS — donde no se explota el trabajo de otras personas, se distribuyen equitativamente los ingresos, se busca producir en armonía con la naturaleza y los vínculos que priman son basados en valores de solidaridad y ayuda mutuase encuentren con un mercado hostil y en donde tienen una difícil participación. A esto se le suma una importante maquinaria publicitaria y de marketing que durante largos años construyó un consumidor que se encuentra, en muchos casos, incapaz de ponderar sus compras más allá del precio, la marca y la comodidad a la que está habituado.

José Luis Coraggio explica esta idea de forma muy clara en su artículo Economía social: conceptos y prácticas:

"En una economía de mercado, donde predominan los valores y las instituciones propias del mercado, tener en cuenta a la sociedad o ser solidario es un desvalor (...). Si para ganar en la competencia hay que bajar costos y esto implica contaminar fuentes de agua, aumentar la productividad a costa de la fertilidad de la tierra, eludir impuestos o salarios legales, o despedir trabajadores, eso es lo que la ética del mercado indica que hay que hacer (26 de septiembre de 2013)."

Es aquí donde identificamos el desafío comunicacional, por una parte, y el de la institucionalización, por otra. 
G $\mathbf{6}$
la ESS es una herramienta sumamente
importante para el desarrollo local y
comunitario que garantiza el empleo digno,
fomenta actividades económicas bajo
relaciones equitativas y democráticas en
el marco del respeto por el medio ambiente
¿Cómo hacer para que este potencial de trabajo y organización, que corre el foco del afán de lucro como única motivación y vuelve a poner en el centro al ser humano para la reproducción de la vida como objetivo esencial de la economía, tenga más presencia en el sentido común de nuestra población? ¿Cómo hacer para avanzar en la construcción de una ciudadanía crítica que sea capaz de reconocer el aporte diferencial que tiene la ESS para la construcción de un mundo mejor y ponderar estos argumentos en su instancia de consumo cotidiano?

En tanto, en el plano de la institucionalización, advertimos que con la recuperación del rol del Estado desde el año 2003 se ha avanzado mucho en materia de ESS y se ha apoyado, fortalecido y otorgado oxígeno a gran cantidad de experiencias que anteriormente eran sostenidas en total soledad por organizaciones sociales, cooperativas, productores asociados, etc. Todas las políticas públicas, programas y leyes existentes dan cuenta de ello. Gracias a eso, el escenario que tenemos hoy y por delante no es el mismo que entonces. En este sentido, el desafío de la institucionalización es trabajar para garantizar que toda esta diversidad de experiencias, en todas sus expresiones, pasen de la legitimidad a la legalidad a través de la construcción de marcos normativos y legales que atiendan y den cuenta de las particularidades de los sujetos protagonistas de la ESS y que, reconociendo el significativo aporte que éstos hacen para el desarrollo de nuestras comunidades, generen escenarios cada vez más favorables para su desenvolvimiento.

Por su capital simbólico, su legitimidad social, su incidencia en la comunidad, su capacidad de formación de cuadros técnicos y de construcción de conocimiento, así como por un ineludible rol de compromiso social, la universidad tiene la posibilidad de hacer grandes aportes en el plano comunicacional y en el de la institucionalización de la ESS. El mayor desafío es que todos los abordajes sean hechos desde la perspectiva de la integralidad.

\section{Conclusiones}

La ESS es una herramienta sumamente importante para el desarrollo local y comunitario que garantiza el empleo digno, fomenta actividades económicas bajo relaciones equitativas y democráticas en el marco del respeto por el medio ambiente. La universidad está llamada, a través de sus tres funciones esenciales, extensión, investigación y docencia, a comprometerse y preocuparse por el desarrollo de la comunidad en la cual está inserta. De este modo, en tanto es una institución estratégica en el desarrollo de la sociedad, como formadora de profesionales, generadora de conocimiento $\mathrm{y}$, a su vez, como actor del desarrollo socioproductivo, la ESS debe ser un eje prioritario para su trabajo con la certeza de que, de este modo, se está aportando a una sociedad más justa, soberana e igualitaria.

La buena noticia es que durante los últimos años la ESS ha comenzado a ganar cada vez más lugar en la agenda pública. Aún falta mucho por hacer y por aprender, pero estamos en camino. Sabemos, eso sí, que otra economía es posible...

\section{Referencias bibliográficas}

Coraggio, J.L. (26 de septiembre de 2013). Economía social: conceptos y prácticas. Página12.

Tomassino, H. y Rodríguez, N. (2010). Tres tesis básicas sobre extensión y prácticas integrales en la Universidad de la República. En Cuadernos de Extensión - No 1 / Integralidad: tensiones y perspectivas (22-23). Uruguay: Cuadernos. Universidad de la República. 